\title{
A Multiparameter Approach to Evaluate Post-Stroke Patients: An Application on Robotic Rehabilitation
}

\author{
Antonella Belfatto ${ }^{1}\left(\mathbb{D}\right.$, Alessandro Scano ${ }^{2, *} \mathbb{C}$, Andrea Chiavenna ${ }^{2}$, Alfonso Mastropietro ${ }^{1}(\mathbb{D}$, \\ Simona Mrakic-Sposta ${ }^{1}$,, Simone Pittaccio ${ }^{3}$, Lorenzo Molinari Tosatti ${ }^{2}$, Franco Molteni ${ }^{4}$ \\ and Giovanna Rizzo ${ }^{1}$
}

1 Institute of Bioimaging and Molecular Physiology (IBFM), National Research Council of Italy (CNR), Via Fratelli Cervi, 93, 20090 Segrate, Italy; belfatto.antonella@gmail.com (A.B.); alfonso.mastropietro@ibfm.cnr.it (A.M.); simona.mrakic@ibfm.cnr.it (S.M.-S.); giovanna.rizzo@ibfm.cnr.it (G.R.)

2 Institute of Intelligent Industrial Technologies and Systems for Advanced Manufacturing (STIIMA), National Research Council of Italy (CNR), Via Alfonso Corti 12, 20133 Milan, Italy; chiavenna.andrea@gmail.com (A.C.); lorenzo.molinaritosatti@stiima.cnr.it (L.M.T.)

3 Institute of Condensed Matter Chemistry and Technologies for Energy, (ICMATE), National Research Council of Italy (CNR), Via Previati 1/E, 23900 Lecco, Italy; simone.pittaccio@cnr.it

4 Rehabilitation Presidium of Valduce Ospedale Villa Beretta, Via Nazario Sauro 17, 23845 Costa Masnaga, Italy; fmolteni@valduce.it

* Correspondence: alessandro.scano@stiima.cnr.it; Tel.: +39-380466-6915

Received: 26 October 2018; Accepted: 11 November 2018; Published: 14 November 2018

\begin{abstract}
Multidomain instrumental evaluation of post-stroke chronic patients, coupled with standard clinical assessments, has rarely been exploited in the literature. Such an approach may be valuable to provide comprehensive insight regarding patients' status, as well as orienting the rehabilitation therapies. Therefore, we propose a multidomain analysis including clinically compliant methods as electroencephalography (EEG), electromyography (EMG), kinematics, and clinical scales. The framework of upper-limb robot-assisted rehabilitation is selected as a challenging and promising scenario to test the multi-parameter evaluation, with the aim to assess whether and in which domains modifications may take place. Instrumental recordings and clinical scales were administered before and after a month of intensive robotic therapy of the impaired upper limb, on five post-stroke chronic hemiparetic patients. After therapy, all patients showed clinical improvement and presented pre/post modifications in one or several of the other domains as well. All patients performed the motor task in a smoother way; two of them appeared to change their muscle synergies activation strategies, and most subjects showed variations in their brain activity, both in the ipsi- and contralateral hemispheres. Changes highlighted by the new multiparametric instrumental approach suggest a recovery trend in agreement with clinical scales. In addition, by jointly demonstrating lateralization of brain activations, changes in muscle recruitment and the execution of smoother trajectories, the new approach may help distinguish between true functional recovery and the adoption of suboptimal compensatory strategies. In the light of these premises, the multi-domain approach may allow a finer patient characterization, providing a deeper insight into the mechanisms underlying the relearning procedure and the level (neuro/muscular) at which it occurred, at a relatively low expenditure. The role of this quantitative description in defining a personalized treatment strategy is of great interest and should be addressed in future studies.
\end{abstract}

Keywords: chronic stroke; robotic assistance; EEG; ERD maps; muscle synergies; synergies clustering; kinematics; Fugl-Meyer Assessment 


\section{Introduction}

Stroke is the leading cause of acquired long-term disability [1]. Among stroke survivors, $25 \%$ to $75 \%$ require help or are fully dependent in activities of daily living [2]. The acute phase following the event requires prompt and specialized care to minimize the impairment to both the somatosensory and motor areas. Nevertheless, the chronic phase may benefit from rehabilitation as well [3-5]. The rehabilitation after stroke is not guided by an established standard protocol; therefore, treatment programs vary in duration, intensity, and frequency [6].

A key factor in the rehabilitation process is the evaluation of the improvements gained during the therapy. The most widely used methods of assessment are clinical scales (physical/cognitive tests or self-reported questionnaires). Some of the most exploited and reliable clinical scales in stroke are the Fugl-Meyer Assessment (FMA, [7]), the Wolf Motor Function Test (WMFT, [8]), and the Motor Activity Log-30 (MAL, [9]). Despite requiring little effort, in terms of operators and time, and providing a wide range of measures, they have some limitations, including lack of sensitivity and specificity along with their operator-dependency [10]. Thus, clinical scales can be integrated with specific neurological assessments, in order to provide an even more detailed portrait of the clinical status of patients [11].

Many instrumental approaches can be employed for this purpose, including kinematics, electromyography (EMG), and brain activity analysis. Each of them investigates a different domain of the hierarchical organization of the neuro-musculoskeletal system.

The effects of rehabilitation on the kinematics of the patient is usually evaluated in terms of articular range of motion, and motor control features such as movement smoothness for the upper limb, or gait biomechanics for the lower limb [12]. These kinematics parameters can be assessed by means of motion tracking systems, such as marker-based optoelectronic devices. Recent works employed kinematics for the assessment of the effects of rehabilitative therapies. Thrane et al. [12,13] related kinematics parameters to clinical scales to observe correlations between the two assessments in neurological patients. The effects of robotic therapies were observed as well, in terms of smoothness, accuracy, and velocity of motor performance, suggesting the possibility of promoting neuroplasticity at both shoulder and elbow level [14]. In a recent study [15] combined constrained induced therapy and robotic therapy demonstrating therapeutic effects on proximal and distal kinematics on children. Kinematics is also used to evaluate motor control capability and smoothness, often coupled with standard clinical tests [16].

As to the quantitative assessment of the muscle activity, a valuable approach for the analysis of EMG signals is the muscle synergy framework. It relies on the hypothesis that the Central Nervous System (CNS) solves the motor control problem by exploiting a limited number of muscle modules (synergies), i.e., coordinated activation patterns of groups of muscles that share a common control signal [17]. Thus, according to this analysis, the CNS uses a limited set of spatial synergies, modulated by a signal identifying their activation level along time while performing a specific task or group of tasks [18]. According to previous studies, in mild-impaired stroke survivors, an altered recruitment pattern can be found, while synergies composition is preserved [19]. In more compromised patients, synergies appear to modify their spatial components, by splitting or merging different motor modules [20]. In a recent study [21], it was found that spatial synergies may be partially related to the patient motor functionality, in a small population of post-stroke patients.

Regarding the activation of the cerebral areas deputed to movement, these can be investigated by means of several methods. Functional magnetic resonance (fMRI) and electroencephalography (EEG) are among the most promising, as shown by the wide literature on the topic [22-25]. In particular, EEG is a low cost, noninvasive, and versatile technique, suitable for measuring brain activity even outdoor and in real-life conditions [26]. EEG highlights coordinated neural activity in specific frequency bands and is very sensitive in detecting stroke-related abnormalities. It is known that a paced event causes an event-related desynchronization (ERD), i.e., "a short-lasting and localized amplitude decrease of rhythmic activity" [23]. A possible indicator of the patient condition is the ERD lateralization (LC), which might also account for the lesion location [27,28]. 
Each of the proposed methodologies investigates a specific domain of the hierarchical organization of the neuro-muscle-skeletal system. Therefore, each methodology provides a detailed but sectorial assessment. Conversely, merging all the domains may provide a comprehensive framework for a quantitative patient profiling, as proposed by preliminary pioneeristic studies [29]. The aim of this paper is to apply the combined assessment on a cohort of five post-stroke chronic patients to evaluate the treatment outcome in a robot-assisted upper limb rehabilitation scenario.

\section{Materials and Methods}

\subsection{Study Design}

A synthetic overview of the study is available in Figure 1, and detailed in this section.

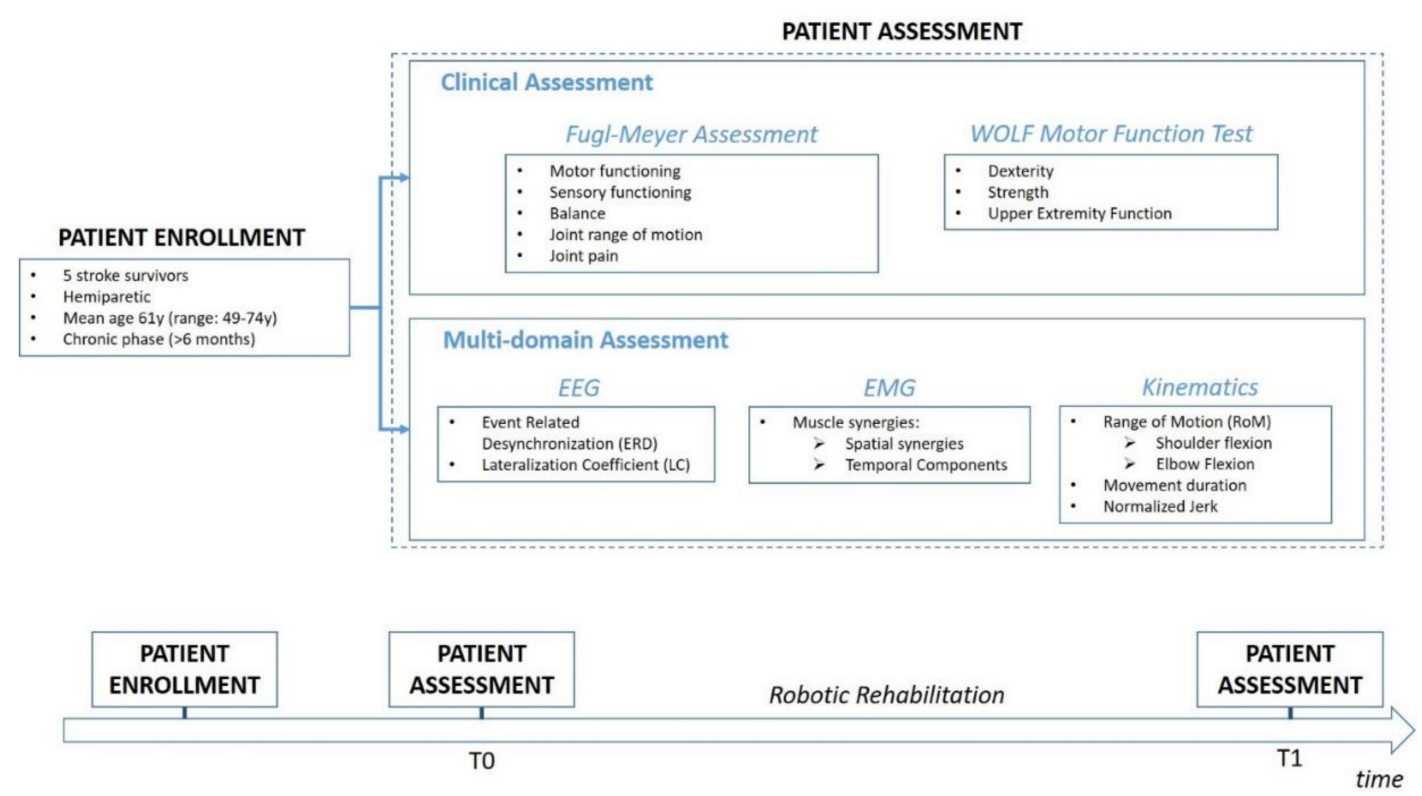

Figure 1. Flow-chart of the study design. Five post-stroke survivors were enrolled in this study (for inclusion criteria and their data see Section 2.1.2). Each patient was administered 1 month of robotic therapy. At T0 (before the therapy) and at T1 (after the therapy), a multidomain assessment that included electroencephalography (EEG) recordings (during passive assisted forearm elbow-flexion), electromyography (EMG), and kinematics recordings (during non-assisted hand-to-mouth movements (HTMM)) was performed. The instrumental evaluations were coupled with standard clinical assessments (Fugl-Meyer Assessment and Wolf Motor Function Test) for a detailed characterization of the effects of the therapy.

\subsubsection{Setting}

The study took place in the Robotic Laboratory, held by the Consiglio Nazionale delle Ricerche at the Presidio di Riabilitazione dell'Ospedale Valduce Villa Beretta, Costa Masnaga (LC), Italy.

\subsubsection{Participants}

Five post-stroke patients (Pt 1-Pt 5, age $61 \pm 11$ years, all male) in the chronic phase of the disease (more than six months from the stroke event) were enrolled. All patients presented upper limb residual impairment. Exclusion criteria were severe upper limb spasticity (Modified Ashworth Scale score $>3$ ) and other concomitant pathologies of the upper limb. Written informed consent was obtained from each subject before inclusion in the study. The study was reviewed and approved by the local Ethics Committee at A. Manzoni Hospital, Lecco, and was conducted in compliance with the Declaration of Helsinki. 


\subsubsection{Robotic Therapy: Equipment and Motor Task}

The rehabilitation was performed using a Mitsubishi Pa10-7 robot, for the execution of 3D movements. Virtual safety walls were implemented to protect the user from improper contact with the robot. A force/torque sensor was located on the handle provided information to a graphic user interface displaying in real time the forces exerted by the user and the position of the handle in the workspace. The task selected for the investigation of the kinematics and of the muscle synergies was the hand-to-mouth movement (HTMM). The trajectory was recorded in manual guidance, performed by an expert clinician, as in the protocol depicted in a previous study [30]. The HTMM trajectory was predetermined and the patient was unable to modify it during the motor execution. The movement standardization allowed the severely impaired patients to perform the task using the whole range of motion thanks to the robot-assistance.

The therapy consisted of 12 sessions of robot-assisted movements ( 3 sessions per week), each one lasting $40 \mathrm{~min}$ that comprehended the execution of point-to-point reaching movements and hand-to-mouth movements. Before and after the therapy (labelled T0 and T1, respectively), a clinical scales-based evaluation and the new multi-parameter assessment were performed for a detailed characterization of each patient' status, as synthetically displayed in Figure 1.

\subsection{Patient Clinical Assessment}

A physiotherapist evaluated each subject at T0 and T1. Two paradigmatic clinical scales were chosen for the evaluation:

- Fugl-Meyer Assessment (FMA), sections A-D (score 0-66) for the measurement of the Body Function domain of the International Classification of Functioning. FMA is a 3-point ordinal scale which evaluates active movement of every upper limb joint and the coordination during motion [7]. It is one of the most widely adopted in the evaluation of the stroke patients [31], has good psychometric measures, such as excellent test-retest reliability [32] and excellent inter-rater/intra-rater reliability [33], and for this reason is highly recommended for use in clinical practice [34].

- Wolf Motor Function Test (WMFT) for the measurement of the Activity domain, especially the capacity qualifier [8]. It consists of 17 items divided in 15 function-based tasks and 2 strength-based tasks. For the function-based tasks, execution time is recorded and the score is given on a 6-point ordinal scale. Together with the Action Research Arm Test, the WMFT is considered the most reliable outcome measure for Activity domain due to its psychometric properties and clinical usefulness [34].

Statistical differences in the pre vs. post values distribution was investigated by means of a two-sided Wilcoxon signed ranks test.

\subsection{Patient Multi-Domain Assessment}

\subsubsection{Kinematic and EMG Assessments}

At T0 and T1, an instrumental data recording was performed with 6-infra-red cameras and a BTS Smart-D system, while patients performed HTMM movements.

Upper-limb kinematics was obtained by tracking the position of passive markers placed on D5 and C7 vertebras, acromion, lateral elbow epicondyle and styloid process of the ulna. Electromyography was recorded from eight muscles, namely, upper trapezius, pectoralis major, anterior deltoid, middle deltoid, posterior deltoid, triceps brachii caput medialis, biceps brachii caput longus, and brachioradialis. The sampling rate was $140 \mathrm{~Hz}$ and $1000 \mathrm{~Hz}$ for kinematics and EMG, respectively. Non-assisted movements were chosen in order to evaluate the range of motion, to characterize the physical status of the patient. Moreover, this choice allowed to assess the voluntary strategy (spatial and temporal) of muscle recruiting in a realistic task. 


\section{Kinematics Analysis}

The 3D coordinates of the markers were filtered with a low-pass, 3rd order Butterworth filter, with a cut-off frequency set at $6 \mathrm{~Hz}$. They were used to compute movement phases, and only the forward phase, when the subject drew the hand toward the mouth, were analyzed. The following parameters were chosen as a selection of relevant measures based on kinematics:

- mean duration of the forward phase;

- shoulder flexion angle, projected into the sagittal plane;

- elbow flexion angle;

- normalized jerk, as a measure of smoothness, computed according to the formula presented in Teulings et al. [35].

Details of the kinematic protocol are available in a previous paper [21].

EMG Analysis and Synergies Extraction

Consistently with kinematic analysis, only forward phases were considered for EMG processing. Each signal from the 8 channels was high-pass filtered with a cut off frequency of $50 \mathrm{~Hz}$, rectified and low-pass filtered at $10 \mathrm{~Hz}$ by means of a Butterworth filter (5th order). Removing low frequencies avoids motion artifacts, while the low pass filter allowed to remove noise and obtain EMG envelopes. Afterwards, all envelopes were aligned and resampled (1000 samples), so that they could be directly compared.

Defining the number of patients in the study as $n P t=5$, the number of experimental conditions (pre and post therapy) as $c=2$, the resampling size of each phase as rate $=1000$, and the number of considered muscles as $n m=8$. The EMG envelopes from each experimental condition were pooled together in a group of $n P t \times c$ aggregated matrices (each one having size rate $\times n m$ ) and synergies were extracted using the non-negative matrix factorization (NMF) algorithm for the decomposition of each of the aggregated matrices [36]. The NMF outputs two matrices: one represents time-invariant, spatial synergies, while the other represents time-variant activations signals for each synergy. The factorization order $r$, used as input for the NMF, was selected in the range from 1 to 8 . For each $r$, the NMF algorithm was applied 1000 times in order to avoid local minima and the repetition accounting for the higher variance of the signal was chosen as the representative of order $r$. The order representing spatial synergies and temporal components was chosen as the minimum explaining at least 0.80 of the variance of the signal. Further synergies were added to the dataset only if increasing $r$ increased the overall explained variance of at least 0.05 .

\subsubsection{Synergies Evaluation Metrics}

For each of the considered stages of the therapy (T0 and T1) spatial synergies and temporal components were compared to assess if the rehabilitation protocol had induced modifications of the motor modules underlying movement. In order to achieve such comparison, for each patient, muscle synergies extracted at $\mathrm{T} 0$ and $\mathrm{T} 1$ were coupled according to the similarity of their temporal components, assessed with the Pearson's correlation coefficient. Then, comparisons were conducted for each patient, on each synergy couple, on both spatial synergies and temporal components. Spatial synergies at $\mathrm{T} 0$ and $\mathrm{T} 1$ were compared with the dot product between the spatial composition of each pair of muscle synergies, while temporal components at $\mathrm{T} 0$ and $\mathrm{T} 1$ were compared using the Pearson's correlation coefficient for each pair of synergies.

\subsubsection{EEG Acquisition Protocol}

EEG measurements were acquired during passive movements at $\mathrm{T} 0$ and $\mathrm{T} 1$, with the support of an upper-limb orthosis. The orthosis was composed of a light exoskeleton enwrapping the upper limb and ensuring constance of shoulder intrarotation (elbow extension in the sagittal plane) and 
forearm supination $\left(90^{\circ}\right.$ supination). The presence of a sensorized hinge placed in correspondence of the elbow joint allowed elbow flexion and extension. Mechanical stops at $90^{\circ}$ and $135^{\circ}$ flexion allowed standardizing of the maximal passive extension limits during passive elbow mobilization. A software implemented in Labview made it possible to acquire the hinge angle during the EEG measurements, so that actual timing and extents of the elbow movements, as well as the presence of unexpected events (cloni, movement errors, ... ), could be checked during post-processing. The software also counted up to a pre-set number of movement repetitions and, for each repetition, generated timed acoustic cues to alert the operator actuating the passive limb motion.

This protocol was adopted for two main reasons: (1) to standardize movements; (2) to allow severely impaired patients to exploit their maximal joint range of motion (active ranges may be more limited than passive ones). The selection of passive movements is supported by previous studies reporting that passive movements may activate brain areas comparably to (or even more than) the active ones [37-39]. The patients were asked to rest in a comfortable supine position, while an expert operator performed a passive flex-extension of the impaired upper limb by moving the orthosis. The protocol lasted $180 \mathrm{~s}$, each movement was triggered by two acoustic signals (1 s apart) identifying the starting time for the arm extension and flexion, respectively. Therefore, the nominal length of a complete movement was $2 \mathrm{~s}$, and an $8 \mathrm{~s}$ break was allowed between repetitions (total time: $10 \mathrm{~s}$ ). The EEG acquisition was performed by means of a cap employing $64 \mathrm{Ag} / \mathrm{AgCl}$ monopolar electrodes placed on the scalp as defined by the International 10/20 system. Impedances were kept below $5 \mathrm{k} \Omega$. The online reference electrode was placed between $\mathrm{Cz}$ and $\mathrm{Cpz}$. The Synamps 2/RT EEG system (Neuroscan) sampled the signals at a frequency of $1 \mathrm{kHz}$. A set of 10 EEG acquisitions (5 patients $\times 2$ time points) was considered for the analysis.

\subsubsection{EEG Data Analysis}

Data EEG analysis was performed offline in Matlab (Mathworks, Natick, MA, USA) using the EEGLAB toolbox. After a re-sampling ( $\mathrm{fs}=500 \mathrm{~Hz}$ ) the signals were filtered by means of a bandpass FIR filter $[0.5 \mathrm{~Hz}, 45 \mathrm{~Hz}])$, and re-referenced to the common average reference. The ear channels (M1 and M2), were excluded from further analysis. The movement onsets $(t=0)$ were individuated by the muscular activation using the EMG acquisitions and an in-house algorithm developed in MATLAB allowing the signal epoching. The epochs featuring a signal exceeding $\pm 100 \mu \mathrm{V}$ and the ones affected by noise (visual inspection aided by the epochs removal tool) were rejected. As a result, in between 9 and 18 epochs were analyzed for each subject. The Independent Component Analysis (ICA) using the logistic infomax ICA algorithm, implemented in EEGLAB was performed in order to remove ocular artifacts, muscle artifacts, lost electrode connections, etc. [40]. The selection of the resulting components to be rejected was supported by SASICA, an automated method [41].

Two frequency bands were separately analyzed, namely the alpha $(8-13 \mathrm{~Hz})$ and beta $(13-25 \mathrm{~Hz})$ bands where the modulations in power were high. The relative power (RP) values for each frequency band was calculated after averaging out all the valid epochs of a patient. RP was computed as the summed absolute band-power in a specific instant, with respect to the average power of the same frequency band at the baseline (in our case: [ $-4.5 \mathrm{~s},-2 \mathrm{~s}]$ ) as suggested by Pfurtsheller et al. [22]. Quantitative indices were then computed for the electrodes of the primary motor cortex (C3 and C4). The electrode C 3 was labeled as 'CONTRA' (C4 = 'IPSI') when the patient lesion was located on the left hemisphere, and the movement was performed with the right limb (the lesioned one). Otherwise, the labels were switched. The absolute band-power values were used to calculate the following quantitative indices:

(1) The mean Event Related Desynchronization (ERD) in the window [0.6 s, $1.8 \mathrm{~s}]$ with respect to the baseline $[-4.5 \mathrm{~s},-2 \mathrm{~s}]$. From now on, $\operatorname{ERDc}\left(\mathrm{P}_{j}\right)$ and $\operatorname{ERDi}\left(\mathrm{P}_{j}\right)$ will refer to the average ERD for the $j$-th patient computed for the CONTRA or IPSI electrode, respectively.

(2) The laterality coefficient (LC), which indicates the activation of the lesioned hemisphere with respect to the other according to the following formula [28]: 


$$
L C(P j)=\frac{E R D c(P j)-E R D i(P j)}{E R D c(P j)+E R D i(P j)}
$$

Quantitative values of ERDi, ERDc, and LC were computed only in the central part of the movement (MOV2) in order to cope with uncertainties and to avoid artifacts related to the start/end of task. The laterality index should span from -1 to 1 , representing the complete lateralization toward the ipsi- or contra-lateral hemisphere, respectively. In case a patient shows positive values for the average relative power in the movement window (ERD $>0$ ), we set it to 0 (no desynchronization occurs) when computing the lateralization, for the sake of consistency with LC meaning and to cope with its range limits. All indices are assessed both at $\mathrm{T} 0$ and $\mathrm{T} 1$, to verify whether changes in the brain activity occurred after robotic rehabilitation. Variations of a generic index $\mathrm{X}$ are computed as $\Delta \mathrm{X}=\mathrm{X}(\mathrm{T} 1)-\mathrm{X}(\mathrm{T} 0)$. Average values are calculated and identified by the formalism $\bar{X}$. A two-sided Wilcoxon signed rank test is performed to verify possible distribution differences for EEG-based indices pre/post rehabilitation $(p=0.05)$.

\section{Results}

All patients could perform the complete clinical trial, each one according to his motor functionality. Results presented in Section 3.1 and Section 3.2 are also listed in Table 1.

\subsection{Clinical Assessment Results}

For FMA, all patients gained at least 1 point, with a maximum pre-post difference of 9 points. According to the WMFT scale, every subject reached at least 1 point of improvement, with a maximum difference of 5 points. The mean scores $( \pm$ STD) are increased for both the measures: from $42.4 \pm 19.9$ to $47 \pm 21.0$ points for FMA $(p=0.06)$ and from $52.4 \pm 24.4$ to $56 \pm 24.0$ for WMFT $(p=0.06)$. Clinical scales scores are reported in Table 1 and synthetically described in Figure 2.

Table 1. Summary of the results: clinical scales and multi-domain approach. For each parameter $X$ of each domain the values at $T 0, T 1$ and the difference $\Delta=X(T 1)-X(T 0)$ are reported. As far as the clinical scales are concerned, the variation over the residual recovery potential $\Delta r e s \%=\frac{X(T 1)-X(T 0)}{\max (X)-X(T 0)} \%$ are listed as well. In bold, variables that differ significantly $(p<0.05)$ comparing values at T1 and T0 (two-sided Wilcoxon rank sum test).

\begin{tabular}{|c|c|c|c|c|c|c|c|}
\hline & & & Patient 1 & Patient 2 & Patient 3 & Patient 4 & Patient 5 \\
\hline \multirow{8}{*}{ Clinical scales } & \multirow{4}{*}{$\begin{array}{c}\text { FMA } \\
(0-66)(\text { a. u. })\end{array}$} & T0 & 56.00 & 36.00 & 11.00 & 61.00 & 48.00 \\
\hline & & $\mathrm{T} 1$ & 57.00 & 44.00 & 12.00 & 65.00 & 57.00 \\
\hline & & $\Delta$ & 1.00 & 8.00 & 1.00 & 4.00 & 9.00 \\
\hline & & $\Delta r e s \%$ & 10.00 & 26.67 & 1.82 & 80.00 & 50.00 \\
\hline & \multirow{4}{*}{$\begin{array}{c}\text { WMFT } \\
(0-75) \text { (a. u.) }\end{array}$} & T0 & 66.00 & 47.00 & 12.00 & 71.00 & 66.00 \\
\hline & & $\mathrm{T} 1$ & 70.00 & 51.00 & 16.00 & 72.00 & 71.00 \\
\hline & & $\Delta$ & 4.00 & 4.00 & 4.00 & 1.00 & 5.00 \\
\hline & & $\Delta r e s \%$ & 44.44 & 14.29 & 6.35 & 25.00 & 55.56 \\
\hline \multirow{12}{*}{ Kinematics } & \multirow{3}{*}{ Shoulder Flexion $\left(^{\circ}\right)$} & T0 & 62.82 & 31.36 & 48.50 & 5.55 & 39.51 \\
\hline & & $\mathrm{T} 1$ & 59.04 & 31.80 & 78.75 & 15.68 & 50.25 \\
\hline & & $\Delta$ & -3.78 & 0.44 & 30.25 & 10.13 & 10.73 \\
\hline & \multirow{3}{*}{ Elbow Flexion $\left({ }^{\circ}\right)$} & T0 & 135.99 & 123.14 & 122.63 & 124.24 & 107.36 \\
\hline & & $\mathrm{T} 1$ & 132.62 & 125.07 & 116.74 & 124.64 & 101.05 \\
\hline & & $\Delta$ & -3.37 & 1.93 & -5.88 & 0.41 & -6.31 \\
\hline & \multirow{3}{*}{ Execution Time (s) } & T0 & 1.25 & 1.42 & 2.35 & 1.08 & 2.03 \\
\hline & & $\mathrm{T} 1$ & 1.20 & 1.18 & 2.27 & 1.24 & 1.62 \\
\hline & & $\Delta$ & -0.05 & -0.24 & -0.09 & 0.16 & -0.41 \\
\hline & \multirow{3}{*}{ Jerk $\left(\mathrm{m} / \mathrm{s}^{3}\right)$} & T0 & 80.73 & 126.39 & 332.54 & 143.65 & 290.71 \\
\hline & & $\mathrm{T} 1$ & 60.92 & 71.93 & 248.34 & 81.46 & 163.03 \\
\hline & & $\Delta$ & -19.81 & -54.46 & -84.20 & -62.19 & -127.68 \\
\hline
\end{tabular}


Table 1. Cont.

\begin{tabular}{|c|c|c|c|c|c|c|c|}
\hline & & & Patient 1 & Patient 2 & Patient 3 & Patient 4 & Patient 5 \\
\hline \multirow{4}{*}{ Synergies } & Spatial Synergies & synergy 1 & 0.64 & 0.96 & 0.84 & 0.93 & 0.92 \\
\hline & (Dot Product) (a. u.) & synergy 2 & 0.94 & 0.94 & 0.87 & 0.86 & 0.89 \\
\hline & Temporal Components & synergy 1 & 0.86 & 0.97 & 0.79 & 0.89 & 0.89 \\
\hline & (Correlation), (a. u.) & synergy 2 & 0.61 & 0.87 & 0.56 & -0.15 & 0.86 \\
\hline \multirow{18}{*}{ EEG } & \multirow{3}{*}{ ERDi-alpha (a. u.) } & T0 & -0.28 & -0.05 & -0.30 & -0.10 & 0.17 \\
\hline & & $\mathrm{T} 1$ & -0.38 & -0.18 & -0.31 & -0.16 & -0.46 \\
\hline & & $\Delta$ & -0.10 & -0.13 & -0.02 & -0.06 & -0.46 \\
\hline & \multirow{3}{*}{$\begin{array}{c}\text { ERDc-alpha } \\
\text { (a. u.) }\end{array}$} & T0 & -0.30 & 0.01 & -0.13 & -0.29 & -0.11 \\
\hline & & $\mathrm{T} 1$ & -0.27 & -0.29 & -0.40 & -0.29 & -0.55 \\
\hline & & $\Delta$ & 0.03 & -0.30 & -0.27 & 0.01 & -0.44 \\
\hline & \multirow{3}{*}{$\begin{array}{l}\text { ERDi-beta } \\
\text { (a. u.) }\end{array}$} & T0 & -0.28 & -0.28 & -0.22 & -0.20 & -0.30 \\
\hline & & $\mathrm{T} 1$ & -0.22 & -0.22 & -0.15 & -0.34 & -0.35 \\
\hline & & $\Delta$ & 0.06 & 0.06 & 0.08 & -0.15 & -0.05 \\
\hline & \multirow{3}{*}{$\begin{array}{c}\text { ERDc-beta } \\
\text { (a. u.) }\end{array}$} & T0 & -0.10 & -0.30 & -0.04 & -0.19 & -0.12 \\
\hline & & $\mathrm{T} 1$ & -0.41 & -0.31 & -0.25 & -0.16 & -0.52 \\
\hline & & $\Delta$ & -0.32 & -0.01 & -0.21 & 0.04 & -0.39 \\
\hline & \multirow{3}{*}{$\begin{array}{l}\text { LC-alpha } \\
\text { (a. u.) }\end{array}$} & T0 & 0.02 & -1.00 & -0.40 & 0.48 & 1.00 \\
\hline & & $\mathrm{T} 1$ & -0.18 & 0.22 & 0.11 & 0.28 & 0.09 \\
\hline & & $\Delta$ & -0.20 & 1.22 & 0.51 & -0.20 & -0.91 \\
\hline & \multirow{3}{*}{$\begin{array}{l}\text { LC-beta } \\
\text { (a. u.) }\end{array}$} & T0 & -0.48 & 0.03 & -0.72 & -0.01 & -0.42 \\
\hline & & $\mathrm{T} 1$ & 0.31 & 0.17 & 0.25 & -0.38 & 0.19 \\
\hline & & $\Delta$ & 0.79 & 0.13 & 0.97 & -0.36 & 0.62 \\
\hline
\end{tabular}

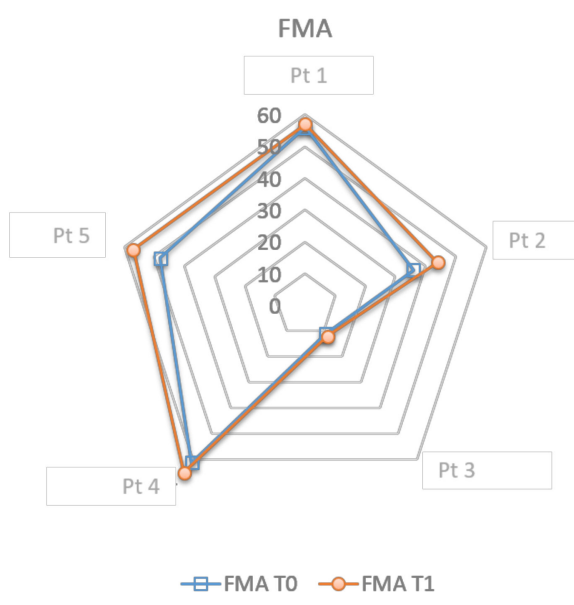

(A)

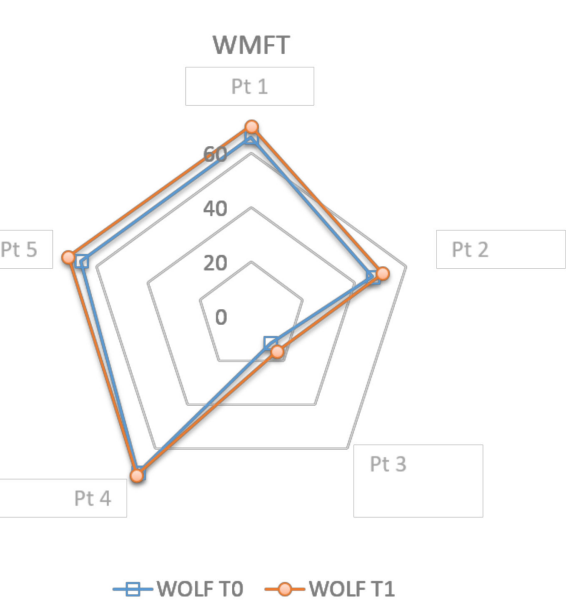

(B)

Figure 2. Radar plots of the patients' scores for the two clinical scales (FMA, (A); WMFT, (B)) at T0 (blue line) and T1 (red line). FMA, Fugl-Meyer Assessment; WMFT, Wolf Motor Function Test.

\subsection{Multi-Domain Assessment Results}

\subsubsection{Kinematics Analysis}

For kinematic analysis, trajectories were expressed in articular body coordinates (shoulder flexion angle and elbow flexion angle), considering the mean achieved by patients during the robotic movements. The mean angles displayed in Figure 3 (right column) were considered at the end of the forward phase when the robot-assisted gesture has just been completed. Averaging across patients, the corresponding values were $38.84^{\circ}$ for shoulder flexion and $122.49^{\circ}$ for elbow flexion. During the free movements, the shoulder flexion angle varied among patients from $5.55^{\circ}$ to $62.82^{\circ}$ (mean value $37.55^{\circ}$ ) at $\mathrm{T} 0$, while it varied from $15.68^{\circ}$ to $78.75^{\circ}$ (mean value $47.10^{\circ}$ ) at $\mathrm{T} 1$. Elbow extension ranged from a minimum value of $107.36^{\circ}$ to a maximum of $135.99^{\circ}$ (mean value $122.67^{\circ}$ ) at $\mathrm{T} 0$, and from $101.05^{\circ}$ to $132.62^{\circ}$ (mean value $120.03^{\circ}$ ) at $\mathrm{T} 1$.

Fastest execution time at T0 was $1.08 \mathrm{~s}$ and the slowest was $2.35 \mathrm{~s}$ (mean $1.63 \mathrm{~s}$ ), while at T1 the fastest execution time was $1.18 \mathrm{~s}$ and the slowest was $2.27 \mathrm{~s}$ (mean $1.50 \mathrm{~s}$ ). 
Normalized Jerk scores ranged from 80.73 to 332.54 (mean 194.81) at T0 and from 60.92 to 248.34 (mean 125.14) at T1. Further details can be found in Table 1.

Pre-post differences measured with the Wilcoxon paired signed ranks test resulted significant only for the Jerk parameter, which decreased at T1 $(p<0.05)$, while for all the other parameters no significant difference was found $(p>0.05)$.

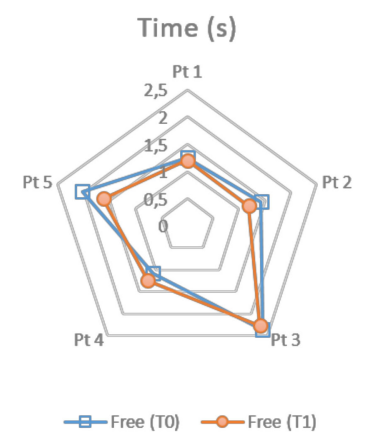

(A)

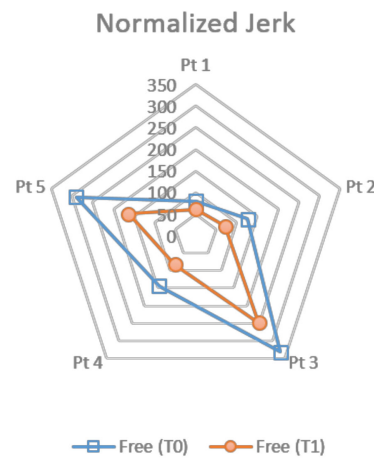

(B)

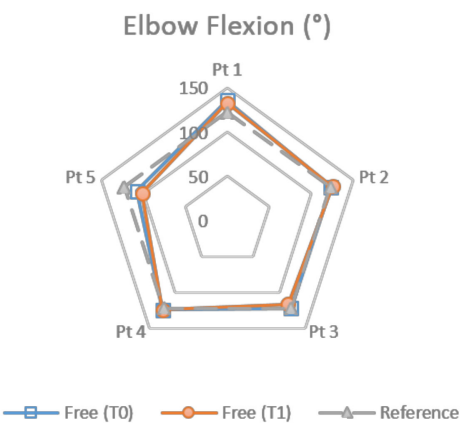

(C)

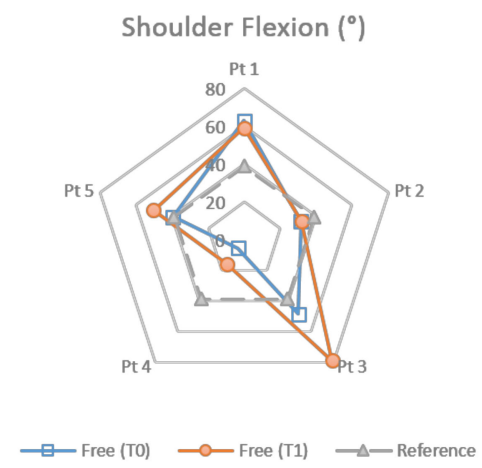

(D)

Figure 3. Radar plots of four kinematics parameters recorded during freely executed HTMM, namely the duration of the forward phase (A), normalized jerk (B), elbow flexion at the end of the movement (C), and shoulder flexion angle (D). For each patient (Pt1, Pt2, Pt3, Pt4 and Pt5) the values at T0 (blue line) and T1 (red line) are shown. For the angles, the reference values (grey dashed line) computed on the trajectory performed by a trained clinician (corresponding to the one imposed by the robot during the rehabilitation), were reported as well.

\subsubsection{EMG (Muscle Synergies) Analysis}

For each patient, at T0 and T1, two main muscle recruitment patterns were found, and will be hereby named "first synergy" and "second synergy". The spatial and temporal components of the first and second synergies at $\mathrm{T} 0$ and $\mathrm{T} 1$ are reported in Figure 4 for all patients. Comparing $\mathrm{T} 0$ and T1, muscle synergies analysis revealed high similarity in spatial synergies both for the first and the second synergy for all the subjects, with a dot product always above 0.84 (with the exception of Pt 1) and a maximum similarity of 0.96 (mean score 0.86 for the first and 0.90 for the second synergy). Temporal components showed a high or very high Pearson's correlation coefficient for the first synergy $(\mathrm{r}>0.79)$, while for the second synergy different correlation values are found, ranging from 0.56 to 0.87 , with a patient presenting even negative correlation $(-0.15)$. 

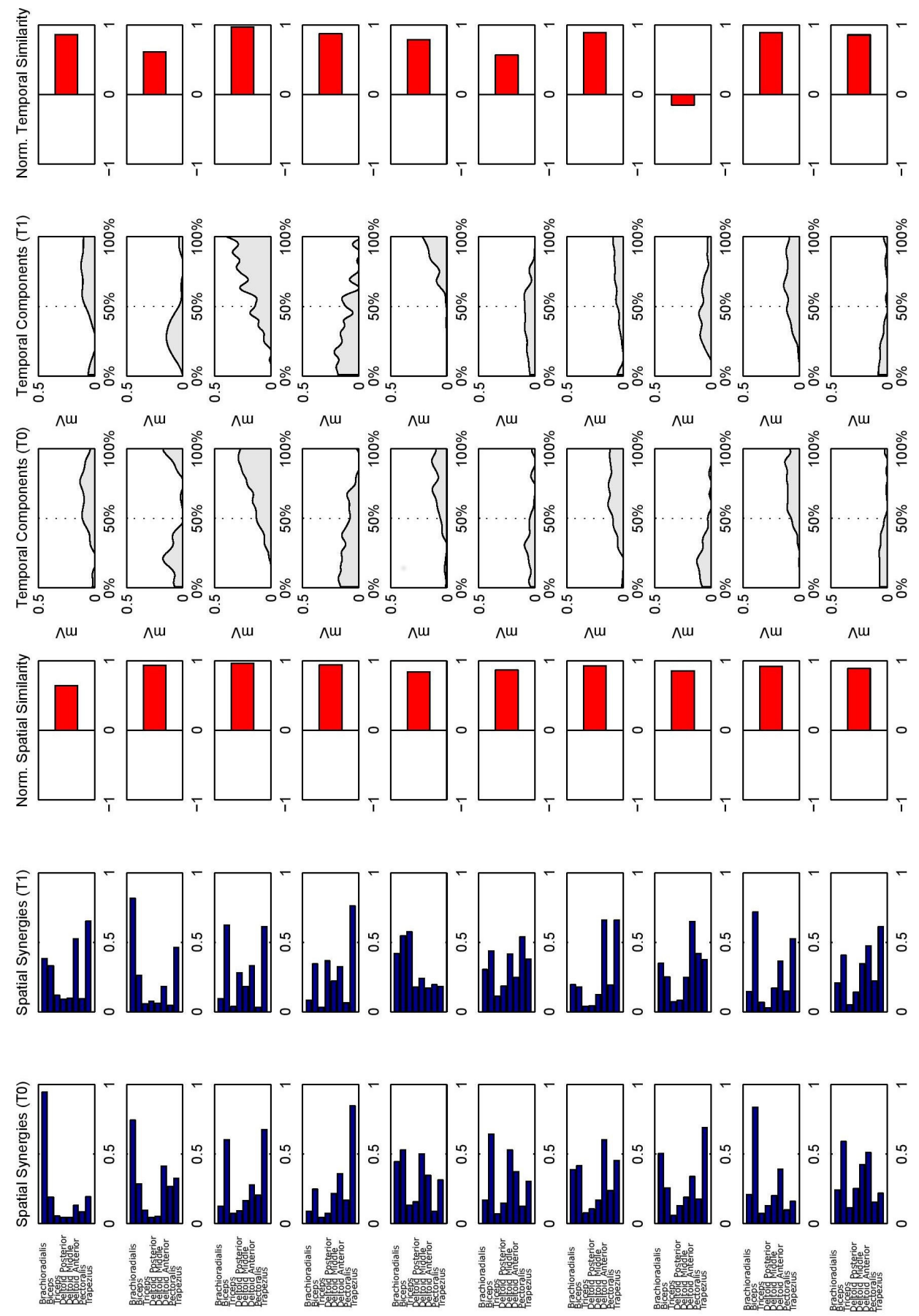

$\begin{array}{lllll}\text { Pt } 1 & \text { Pt } 2 & \text { Pt } 3 & \text { Pt } 4 & \text { Pt } 5\end{array}$

Figure 4. Patients' spatial synergies, extracted at T0 and T1, are depicted in the first and second columns, respectively. The corresponding temporal components are depicted in the fourth and fifth row. For each T0/T1 correspondent couple of motor modules, similarity on spatial synergies and on temporal components is reported in column 3 and column 6, respectively.

\subsubsection{EEG Analysis}

The EEG analysis showed that, on average, a desynchronization was recognizable in primary motor cortex bilaterally (electrodes: C3 and C4), in both the pre- and post-rehabilitation phases as depicted in Figure 5. However, at T0, the ERD appear to be mostly occurring in the beta band for 
the ipsilateral hemisphere, while temporally limited to only part of the movement execution in the contralateral. Conversely, at T1, the desynchronization spreads to all frequencies (alpha and beta) and to the overall movement execution in both ipsilateral and contralateral areas.

In order to provide a comprehensive picture of the overall brain activity, the RP values were computed for all electrodes and averaged out on the five patients by mirroring the results of the task performed with the left hand. The spatial ERD maps, averaged out in 5 times windows, are shown in Figure 6. It can be seen that, despite being spread to most of the scalp, the average desynchronization evolution varies from $\mathrm{T} 0$ to $\mathrm{T} 1$, especially in alpha. While before treatment the ERD starts after the movement initiation (MOV2, MOV3) and is slightly pronounced, after the rehabilitation it occurs even in the PRE-MOV window. Beta band changes were not noticeable comparing T0 to T1.

The T0 and T1 distributions of ERDi, ERDc and LC were not significantly different both for alpha and beta bands. Despite this result, a decreasing trend is evident in the alpha band both for the ipsilateral and contralateral hemispheres as shown in Figure 7, meaning that $\overline{E R D i}(T 1)<\overline{E R D i}(T 0)$ and $\overline{E R D c}(T 1)<\overline{E R D c}(T 0)$, while an average ERD intensification occurred for the contralateral side only in the beta band. In agreement with the previous observations, there is not a clear trend in the alpha-based LC distribution pre-/post-treatment since both ERDi and ERDc decrease, while LC in the beta band moves from negative (T0) to positive (T1) values (ERDc decreades while ERDi seems stable).

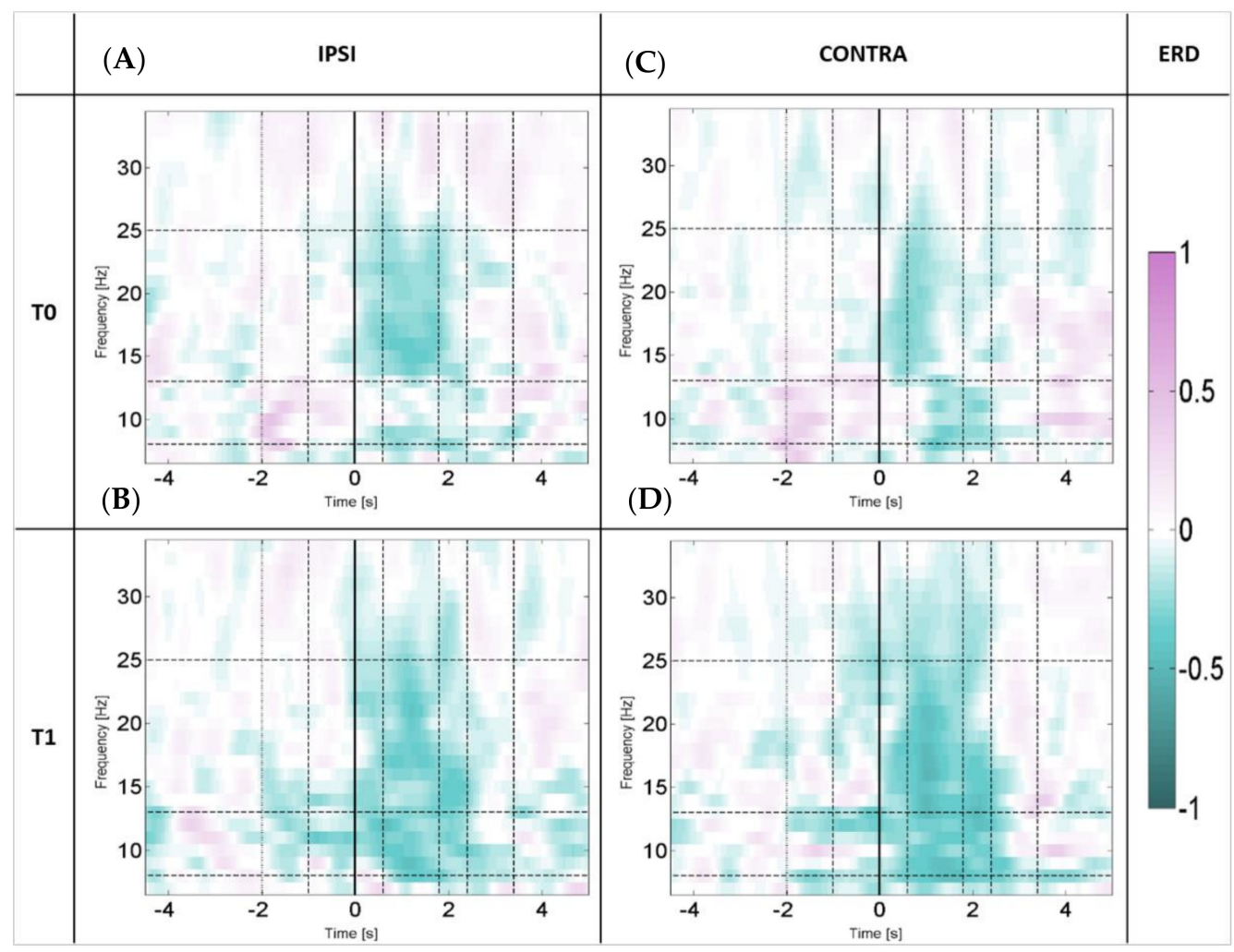

Figure 5. The average maps representing the time-frequency distribution of the ERDi (A,B) and the ERDc (C,D) before treatment (T0, A-C) and after robotic rehabilitation (T1, B-D) are shown. A color legend, on the right of each scheme, identifies the corresponding ERD intensity. The baseline [ $-4.5 \mathrm{~s},-2 \mathrm{~s}]$, the pre-movement $[-1 \mathrm{~s}, 0 \mathrm{~s}]$ the central part of the movement [0.6 s, $1.8 \mathrm{~s}]$ and the post-movement $[2.4 \mathrm{~s}, 3.4 \mathrm{~s}]$ windows along with the alpha $[8 \mathrm{~Hz}-13 \mathrm{~Hz}]$ and beta $[13 \mathrm{~Hz}-25 \mathrm{~Hz}]$ bands, are delimited by dashed lines perpendicular to the $\mathrm{x}$ and $\mathrm{y}$ axis, respectively. 


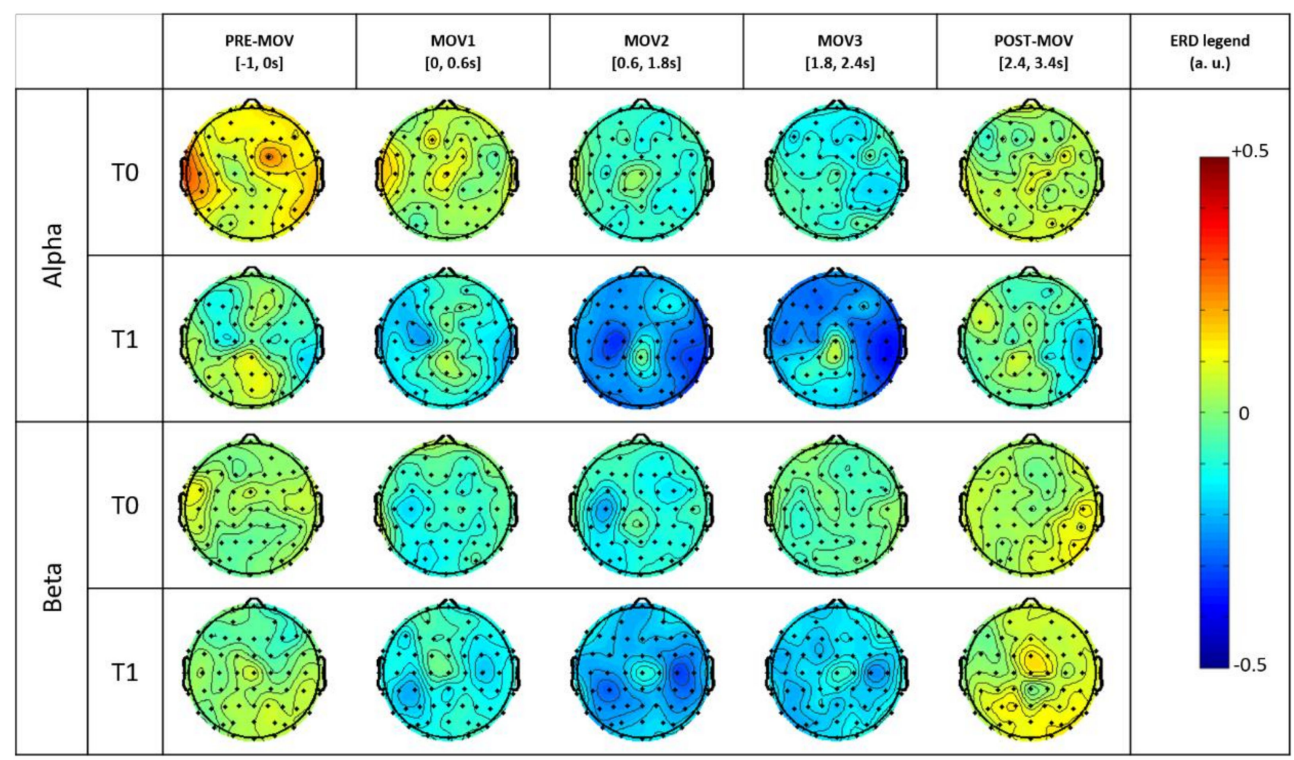

Figure 6. Topographic visualization of the average ERD (arbitrary unit) in five time windows, i.e., before movement initiation (PRE-MOV $[-1,0 \mathrm{~s}]$ ), during movement (MOV1 [0, $0.6 \mathrm{~s}]$, MOV2 [0.6, 1.8 s], MOV3 [1.8, 2.4 s]) and after the movement termination (POST-MOV [2.4, $3.4 \mathrm{~s}]$ ). The maps of patients performing the movement by means of the left hand (impaired one) were mirrored.

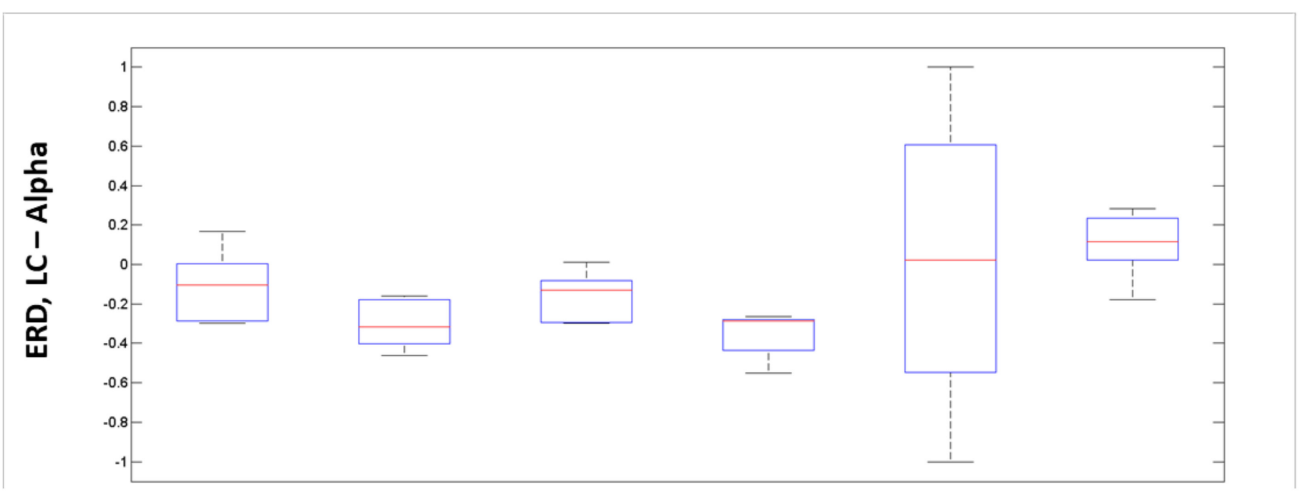

(A)

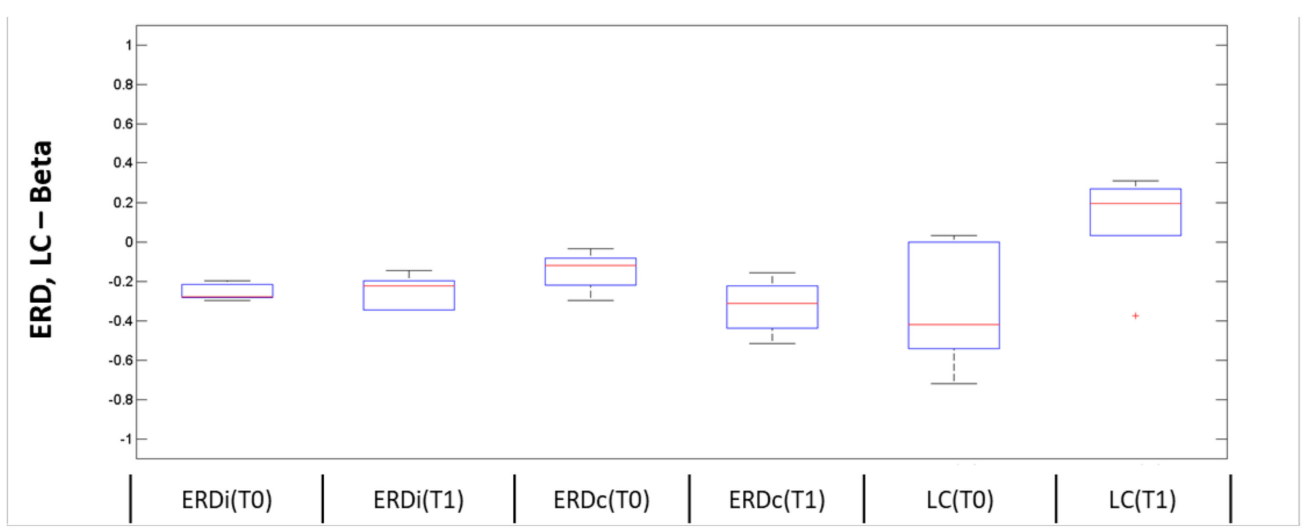

(B)

Figure 7. Distributions of ERDi, ERDc and LC, before (T0) and after (T1) the robotic rehabilitation, are shown for the alpha (A) and beta (B) bands. The central line indicates the median, while the lower and upper extremities of the boxes represent the 25th and 75th percentiles, respectively. The whiskers comprise all the data points distribution except the outliers, which are plotted separately using the '+' mark. 


\section{Discussion}

\subsection{Multi-Domain Assessment}

\subsubsection{Kinematics}

When considering the active range of motion, all the patients could reach the mouth with their impaired limb both at T0 and T1 with exception made for Patient 3. More in detail, elbow flexion is the main biomarker characterizing the capability of performing the HTMM. Therefore, with respect to the reference trajectory, it can be observed that patients' free movement shows similar values in elbow flexion at the end of the movement, indicating that all five patients were able to achieve physiological angles at elbow level, with no noticeable difference between T0 and T1. On the contrary, the shoulder flexion angle is similar at T0 and T1 in Patients 1 and 2, while in Patients 3 and 5 it shows high variability since at $\mathrm{T} 1$ the shoulder flexion detaches more from the reference value in respect to $\mathrm{T} 0$. Finally, Patient 4 shows a slight recovery toward the physiological pattern. Such results may suggest that the effect of the therapy was, on average, not effective on the range of motion, since it did not promote the coordination of articular patterns towards the reference path performed with the robot. This conclusion could also be suggested by the emergence of a compensatory strategy adopted by Patient 3 to reach the mouth by over-flexing the shoulder. However, such findings can be related only to the reduced sample of patients enrolled in this study, and cannot be considered as representative of the post-stroke chronic population.

Despite the fact that the observed changes in execution time are very slight and not statistically significant, the kinematics of the movement at $\mathrm{T} 1$ is characterized by a noteworthy increase in the movement smoothness, which instead is statistically significant. This result is relevant since smooth movement execution is a typical feature of skilled motor control. Thus, even if the cohort of patients is too small to provide generalizable outcomes, the kinematic assessment suggests that relevant processes of motor learning may take place during the robot therapy. Such processes may not be oriented towards the recovery of a reference trajectory, but, rather, more likely to adapting motor learning strategies to compensate for motor impairments, including smoother activations, as already suggested by previous works [42].

\subsubsection{EMG (Muscle Synergies)}

Two main patterns are coordinated for the execution of the HTMM. As explained in Section 2.3.4, for each patient, synergies at $\mathrm{T} 0$ and $\mathrm{T} 1$ were matched according to the similarity of their temporal components. Such method showed that the first extracted synergy is related mainly to the final part of the movement (when the hand is approaching the mouth), while the second synergy is recruited to begin the flexion of the elbow and the shoulder, at the early stages of the movement. Interestingly, the matching of temporal components (pre/post rehabilitation) was always confirmed by temporal high correlations $(>0.79)$ on the first synergy. Good matching is in general found also when considering the corresponding spatial synergies. It can be concluded that on the considered sample of patients, the therapy did not modify sensibly the motor modules underlying the final phase of the movement. On the contrary, the second synergy was modified in temporal recruitment in Patient 4 and, to a less extent, in Patients 1 and 3. This result is also coupled with a very slight modification of the spatial synergies. Overall, these preliminary results suggest that patients who were administered the robot therapy, achieved only a slight reshaping of the available muscle motor modules coordinated for accomplishing the HTMM, but were able instead to modify their temporal recruitment patterns. These results are relevant since they suggest the possibility of promoting slight modifications in chronic patients as shown in the literature for sub-acute stroke patients performing robotic rehabilitation [43]. In such patients, improvement of motor performance due to the treatment was achieved in conjunction with slight modifications of muscle synergies, which appeared to be 
influenced by the different recovering mechanisms across patients presumably due to the heterogeneity of lesions, sides, and location of the accident.

\subsubsection{EEG}

The EEG desynchronization is noticeable in both ipsi- and contra-lateral hemispheres at $\mathrm{T} 0$ and T1 for the beta band; in three cases (patients 1,3 and 4) it is also recognizable for the alpha band at T0. Furthermore, all patients show an ERD post-treatment in the alpha band as well. The literature suggests that the lack of ERD may imply a severe impairment causing a baseline so low that no desynchronization can be identified [44]. The fact that, conversely, all patients show a desynchronization at $\mathrm{T} 1$ can be the first indicator that the treatment obtained some positive effect at a cortical level as well. Moreover, a decreasing trend can be outlined for ERDc both in beta and alpha band while for ERDi the same consideration applies to the alpha band only. Such finding agrees with previous studies suggesting that an enhancement of the desynchronization (especially in the contralateral hemisphere) may reflect a motor recovery [45]. The fact that the desynchronization does not occur symmetrically in the ipsilateral and contralateral side can be thought as another indicator, not only of the effect of the treatment, but also of the recovery strategy adopted by the specific patient at the cortical level. In fact, in the literature, it was shown that healthy subjects often show larger desynchronization in the contralateral side with respect to the limb performing the movement [46,47], therefore a shift toward this physiological condition may suggest an improvement. Concurrently, the ipsilateral region can be used to compensate the lesioned hemisphere [28], possibly due to a reduced interhemispheric inhibition [48]. This can also be seen analyzing the behavior of the LC index (beta) which increases from T0 to T1, moving from a negative (ERDi $<$ ERDc) to a positive (ERDi $>$ ERDc) value in 3 patients out of 5 . The only subject showing an LC decrease is Patient 4 , who also shows the highest motor functionality according to the FMA at T0, and almost a complete recovery of the motor function at T1. The unexpected LC decrease might be associated to an automatization process, which can occur in skilled subjects when repeating a known gesture that requires a reduced effort and consequently a reduced activation of the contralateral side [22,49].

\subsection{Integration}

Investigating different layers of the hierarchy of the neuro-muscle-skeletal system, all the domains appear to be affected by the robotic rehabilitation.

A general agreement between the assessments achieved with kinematics and muscle synergies was noticeable. In fact, as the elbow flexion at the end of the movement is not altered at T1 in respect to $\mathrm{T} 0$, muscle motor patterns at the end of the movement do not appear to be affected by the therapy. Similarly, synergy 2 is altered in some patients, and this finding might be observed on shoulder flexion angle as well. Furthermore, the modifications found in muscle patterns and trajectories may be ascribed to an attempt at executing the movement in a more skilled way, as detected by the smoothness index. Changes in recruitment of synergy 2 are correlated to alterations in the kinematics of the shoulder and movement smoothness. In this view, the robotic therapy was not able to give patients the capability of mimicking a "reference-like" path but helped in coordinating compensatory strategies that allowed the patients to perform the movement with an increased control (decreased jerk). This finding is coherent with previous literature that used f-MRI to highlight that additional recruitment of sensorimotor areas after stroke may not correspond to restitution of motor function, but more likely to adaptive motor learning strategies to compensate for motor impairments [42]. In this study, some of the EEG findings agree as well, suggesting that neuroplastic processes are taking place at the cortical level, as observed in similar studies [50]. In particular, the enhancement of the ERD in the contralateral side, in the alpha band, and the increase of the LC, in the beta band, suggest the possible partial reorganization of the cortical lesioned areas [45], that are reflected, at a lower hierarchical level, by a modified proximal kinematics and, consequently, by slightly altered motor module recruitment patterns. 
It is also interesting to compare standard clinical assessments with the multi-domain approach. However, when trying to match the two approaches, the reduced number of patients and their heterogeneity have to be taken into account. In detail, two out of five patients increased their FMA scores over the threshold of minimal detectable change, which is clinically set between 4.25 and 7.25 points [51]. Patient 3 also shows a noticeable improvement ( +4 points), and due to his high FMA at $\mathrm{T} 0$, he would have had to recover completely to exceed the threshold. The two remaining patients only gained 1 point, thus their clinical status at T1 was not modified from T0. Moreover, the effects of the therapy are not easily quantified since not only patients show different improvements, but they also began the therapy with different clinical conditions.

An instance of the coherency between clinical scales and the multi-parameter assessment can be provided considering Patients 1 and 5 that, starting with different scores at T0, reach the same clinical status at T1 (FMA $=57)$, and thus are more easily comparable than the heterogeneous sample. In these two patients, the equal FMA (T1) is coupled with a very similar score even in the WMFT, suggesting that the status on the body function domain is correlated to the status in the activity domain. This coherence found between clinical scales can be observed also in some domains of the instrumental evaluation. At a kinematic level, both the patients execute the movement similarly, converging towards a slight shoulder compensation, comparable elbow flexion, and an increase of the smoothness of the movement, coupled with a slight reduction in movement execution time. On the contrary, while both patients have alterations in muscle patterns (especially in synergy 2), the more relevant modifications are unexpectedly found in Patient 1 who showed a less noticeable increase in the FMA. Even when considering EEG evaluations, some similarities can be spotted at T1 between the two patients, for instance considering the results in the beta band. It is worth noticing that the patient who started from a more critical clinical status according to FMA (Patient 5) was also the one showing a positive ERDi (alpha) at T0. Some differences can be found in the LC of the alpha waves, in fact, unexpectedly Patient 1 shows at T1 a negative LC (alpha), he is also the one reporting a lower recovery in FMA. It is reasonable to explain the $\mathrm{LC}<0$ with a remapping of the functionality belonging to the lesioned hemisphere (contralateral) on the ipsilateral side, which allows a compensatory motor recovery.

The previous considerations suggest that, in general, kinematics are strongly related to the patient status described by clinical scales, which could be an expected outcome since they measure similar parameters. Instead, EEG variables may be relevant not only for the evaluation of the status of the patients, but even as descriptors or predictors of the possible outcome of the therapy [52]. Moreover, muscle synergies show partial coherency with the kinematics, as observed also in previous works [21], and may reflect at muscle level the alterations found in cortical EEG-based parameters. These observations confirm that the multiparameter approach might be valuable to assess rehabilitation effects from different points of view, allowing the detection of finer alterations that would be missed by standard clinical approaches or mono-parameter analysis. Such observation is in accordance with the results found in chronic stroke patients by Kitago et al. [53] reporting that improvement in the changes in the quality of entire movement trajectories were not accompanied by changes in clinical measures of impairment or function.

In conclusion, the multidomain approach provides results partially consistent with the clinical assessment and might provide useful data to integrate and explain its outcomes. Briefly, the advantages of applying the instrumental assessment can be synthesized in: (1) the recognition of the level at which modifications occurs; (2) deeper insight into the recovery process either toward a physiological condition or by means of compensatory strategies. Therefore, the multidomain approach could be exploited to perform an accurate patient stratification and to provide the best therapeutic plan accordingly.

Author Contributions: A.B. and A.S. analyzed the data, designed and wrote the paper. A.M., S.M.-S., A.C. and S.P. acquired the data, contributed to data analysis, and revised the paper. G.R. conceived the study design, supervised the study, and revised the paper. F.M. recruited the patients, gave the clinical directives, and revised the paper. L.M.T. provided the technological design and revised the paper. 
Funding: This paper was funded by the RIPRENDO@home, a Regional research project funded under the framework agreement between Regione Lombardia and the National Council of Research (D.G.R. n. 3728-11 July 2012) and by Future Home for Future Communities, a Regional research project funded under the framework agreement between Regione Lombardia and the National Council of Research (D.G.R. n. 3866-17 July 2015).

Acknowledgments: The authors wish to acknowledge the Riprendo@Home Research Project.

Conflicts of Interest: The authors declare no conflict of interest.

\section{References}

1. Pollock, A.; Farmer, S.E.; Brady, M.C.; Langhorne, P.; Mead, G.E.; Mehrholz, J.; van Wijck, F. Interventions for improving upper limb function after stroke. Cochrane Database Syst. Rev. 2013. [CrossRef]

2. Miller, E.L.; Murray, L.; Richards, L.; Zorowitz, R.D.; Bakas, T.; Clark, P.; Billinger, S.A. Comprehensive overview of nursing and interdisciplinary rehabilitation care of the stroke patient: A scientific statement from the American Heart Association. Stroke 2010, 41, 2402-2448. [CrossRef] [PubMed]

3. Ang, K.K.; Chua, K.S.; Phua, K.S.; Wang, C.; Chin, Z.Y.; Kuah, C.W.; Low, W.; Guan, C. A randomized controlled trial of EEG-based motor imagery brain-computer interface robotic rehabilitation for stroke. Clin. EEG Neurosci. 2015, 46, 310-320. [CrossRef] [PubMed]

4. Fasoli, S.E.; Krebs, H.I.; Stein, J.; Frontera, W.R.; Hogan, N. Effects of robotic therapy on motor impairment and recovery in chronic stroke. Arch. Phys. Med. Rehabil. 2003, 84, 477-482. [CrossRef] [PubMed]

5. Lloréns, R.; Gil-Gómez, J.A.; Alcañiz, M.; Colomer, C.; Noé, E. Improvement in balance using a virtual reality-based stepping exercise: A randomized controlled trial involving individuals with chronic stroke. Clin. Rehabil. 2015, 29, 261-268. [CrossRef] [PubMed]

6. Lo, A.C.; Guarino, P.D.; Richards, L.G.; Haselkorn, J.K.; Wittenberg, G.F.; Federman, D.G.; Bever, C.T., Jr. Robot-assisted therapy for long-term upper-limb impairment after stroke. N. Engl. J. Med. 2010, 362, 1772-1783. [CrossRef] [PubMed]

7. Fugl-Meyer, A.R.; Jääskö, L.; Leyman, I.; Olsson, S.; Steglind, S. The post-stroke hemiplegic patient. 1. A method for evaluation of physical performance. Scand. J. Rehabil. Med. 1975, 7, 13-31. [PubMed]

8. Wolf, S.L.; Catlin, P.A.; Ellis, M.; Archer, A.L.; Morgan, B.; Piacentino, A. Assessing Wolf motor function test as outcome measure for research in patients after stroke. Stroke 2001, 32, 1635-1639. [CrossRef] [PubMed]

9. Uswatte, G.; Taub, E.; Morris, D.; Vignolo, M.; McCulloch, K. Reliability and validity of the upper-extremity Motor Activity Log-14 for measuring real-world arm use. Stroke 2005, 36, 2493-2496. [CrossRef] [PubMed]

10. Harrison, J.K.; McArthur, K.S.; Quinn, T.J. Assessment scales in stroke: Clinimetric and clinical considerations. Clin. Interv. Aging 2013, 8, 201. [PubMed]

11. Scano, A.; Chiavenna, A.; Malosio, M.; Tosatti, L.M.; Molteni, F. Kinect V2 implementation and testing of the reaching performance scale for motor evaluation of patients with neurological impairment. Med. Eng. Phys. 2018, 56, 54-58. [CrossRef] [PubMed]

12. Ferrarin, M.; Bovi, G.; Rabuffetti, M.; Mazzoleni, P.; Montesano, A.; Moroni, I.; Pareyson, D. Reliability of instrumented movement analysis as outcome measure in Charcot-Marie-Tooth disease: Results from a multitask locomotor protocol. Gait Posture 2011, 34, 36-43. [CrossRef] [PubMed]

13. Thrane, G.; Sunnerhagen, K.S.; Persson, H.C.; Opheim, A.; Alt Murphy, M. Kinematic upper extremity performance in people with near or fully recovered sensorimotor function after stroke. Phys. Theory Pract. 2018, 1-11. [CrossRef] [PubMed]

14. Pila, O.; Duret, C.; Gracies, J.M.; Francisco, G.E.; Bayle, N.; Hutin, É. Evolution of upper limb kinematics four years after subacute robot-assisted rehabilitation in stroke patients. Int. J. Neurosci. 2018, 1-10. [CrossRef] [PubMed]

15. Beretta, E.; Cesareo, A.; Biffi, E.; Schafer, C.; Galbiati, S.; Strazzer, S. Rehabilitation of Upper Limb in Children with Acquired Brain Injury: A Preliminary Comparative Study. J. Healthc. Eng. 2018. [CrossRef] [PubMed]

16. Carpinella, I.; Cattaneo, D.; Ferrarin, M. Quantitative assessment of upper limb motor function in Multiple Sclerosis using an instrumented Action Research Arm Test. J. Neuroeng. Rehabil. 2014, 11, 67. [CrossRef] [PubMed]

17. Bizzi, E.; Cheung, V.C. The neural origin of muscle synergies. Front. Comput. Neurosci. 2013, 7, 51. [CrossRef] [PubMed] 
18. D'Avella, A.; Portone, A.; Fernandez, L.; Lacquaniti, F. Control of fast-reaching movements by muscle synergy combinations. J. Neurosci. 2006, 26, 7791-7810. [CrossRef] [PubMed]

19. Cheung, V.C.; Piron, L.; Agostini, M.; Silvoni, S.; Turolla, A.; Bizzi, E. Stability of muscle synergies for voluntary actions after cortical stroke in humans. Proc. Natl. Acad. Sci. USA 2009, 106, 19563-19568. [CrossRef] [PubMed]

20. Cheung, V.C.; Turolla, A.; Agostini, M.; Silvoni, S.; Bennis, C.; Kasi, P.; Bizzi, E. Muscle synergy patterns as physiological markers of motor cortical damage. Proc. Natl. Acad. Sci. USA 2012, 109, 14652-14656. [CrossRef] [PubMed]

21. Scano, A.; Chiavenna, A.; Malosio, M.; Molinari Tosatti, L.; Molteni, F. Muscle Synergies-Based Characterization and Clustering of Poststroke Patients in Reaching Movements. Front. Bioeng. Biotechnol. 2017, 5, 62. [CrossRef] [PubMed]

22. Pfurtscheller, G.; Da Silva, F.L. Event-related EEG/MEG synchronization and desynchronization: Basic principles. Clin. Neurophys. 1999, 110, 1842-1857. [CrossRef]

23. Pfurtscheller, G. Functional brain imaging based on ERD/ERS. Vis. Res. 2001, 41, 1257-1260. [CrossRef]

24. Attwell, D.; Iadecola, C. The neural basis of functional brain imaging signals. Trends Neurosci. 2002, 25, 621-625. [CrossRef]

25. Henson, R.N.; Williams, N. Recent Advances in Functional Neuroimaging Analysis for Cognitive Neuroscience; University of Cambridge: Cambridge, UK, 2018.

26. Debener, S.; Minow, F.; Emkes, R.; Gandras, K.; Vos, M. How about taking a low-cost, small, and wireless EEG for a walk? Psychophysiology 2012, 49, 1617-1621. [CrossRef] [PubMed]

27. Kaiser, V.; Daly, I.; Pichiorri, F.; Mattia, D.; Müller-Putz, G.R.; Neuper, C. Relationship between electrical brain responses to motor imagery and motor impairment in stroke. Stroke 2012, 43, 2735-2740. [CrossRef] [PubMed]

28. Park, W.; Kwon, G.H.; Kim, Y.H.; Lee, J.H.; Kim, L. EEG response varies with lesion location in patients with chronic stroke. J. Neuroeng. Rehabil. 2016, 13, 21. [CrossRef] [PubMed]

29. Bulea, T.C.; Kilicarslan, A.; Ozdemir, R.; Paloski, W.H.; Contreras-Vidal, J.L. Simultaneous scalp electroencephalography (EEG), electromyography (EMG), and whole-body segmental inertial recording for multi-modal neural decoding. J. Vis. Exp. 2013, 77. [CrossRef] [PubMed]

30. Caimmi, M.; Chiavenna, A.; Scano, A.; Gasperini, G.; Giovanzana, C.; Molinari, L.T.; Molteni, F. Using robot fully assisted functional movements in upper-limb rehabilitation of chronic stroke patients: Preliminary results. Eur. J. Phys. Rehabil. Med. 2017, 53, 390-399. [PubMed]

31. Santisteban, L.; Térémetz, M.; Bleton, J.P.; Baron, J.C.; Maier, M.A.; Lindberg, P.G. Upper limb outcome measures used in stroke rehabilitation studies: A systematic literature review. PLoS ONE 2016, 11, e0154792. [CrossRef] [PubMed]

32. Platz, T.; Pinkowski, C.; van Wijck, F.; Kim, I.H.; Di Bella, P.; Johnson, G. Reliability and validity of arm function assessment with standardized guidelines for the Fugl-Meyer Test, Action Research Arm Test and Box and Block Test: A multicentre study. Clin. Rehabil. 2005, 19, 404-411. [CrossRef] [PubMed]

33. Duncan, P.W.; Propst, M.; Nelson, S.G. Reliability of the Fugl-Meyer assessment of sensorimotor recovery following cerebrovascular accident. Phys. Ther. 1983, 63, 1606-1610. [CrossRef] [PubMed]

34. Murphy, M.A.; Resteghini, C.; Feys, P.; Lamers, I. An overview of systematic reviews on upper extremity outcome measures after stroke. BMC Neurol. 2015, 15, 29. [CrossRef] [PubMed]

35. Teulings, H.L.; Contreras-Vidal, J.L.; Stelmach, G.E.; Adler, C.H. Parkinsonism reduces coordination of fingers, wrist, and arm in fine motor control. Exp. Neurol. 1997, 146, 159-170. [CrossRef] [PubMed]

36. Lee, D.D.; Seung, H.S. Algorithms for non-negative matrix factorization. In Advances in Neural Information Processing Systems; The MIT Press: Cambridge, MA, USA, 2001; pp. 556-562.

37. Pittaccio, S.; Garavaglia, L.; Molteni, E.; Guanziroli, E.; Zappasodi, F.; Beretta, E.; Strazzer, S.; Molteni, F.; Villa, E.; Passaretti, F. Can passive mobilization provide clinically-relevant brain stimulation? A pilot EEG and NIRS study on healthy subjects. In Proceedings of the 2013 35th Annual International Conference of the IEEE Engineering in Medicine and Biology Society (EMBC), Osaka, Japan, 3-7 July 2013; pp. 3547-3550.

38. Kang, H.; Park, W.; Kang, J.H.; Kwon, G.H.; Kim, S.P.; Kim, L. A neural analysis on motor imagery and passive movement using a haptic device. In Proceedings of the 2012 12th International Conference on Control, Automation and Systems, JeJu Island, Korea, 17-21 October 2012; pp. 1536-1541. 
39. Kaiser, V.; Kreilinger, A.; Müller-Putz, G.R.; Neuper, C. First steps toward a motor imagery based stroke BCI: New strategy to set up a classifier. Front. Neurosci. 2011, 5, 86. [CrossRef] [PubMed]

40. Delorme, A.; Makeig, S. EEGLAB: An open source toolbox for analysis of single-trial EEG dynamics including independent component analysis. J. Neurosci. Methods 2004, 134, 9-21. [CrossRef] [PubMed]

41. Chaumon, M.; Bishop, D.V.; Busch, N.A. A practical guide to the selection of independent components of the electroencephalogram for artifact correction. J. Neurosci. Methods 2015, 250, 47-63. [CrossRef] [PubMed]

42. Buma, F.E.; van Kordelaar, J.; Raemaekers, M.; van Wegen, E.E.; Ramsey, N.F.; Kwakkel, G. Brain activation is related to smoothness of upper limb movements after stroke. Exp. Brain Res. 2016, 234, 2077-2089. [CrossRef] [PubMed]

43. Tropea, P.; Monaco, V.; Coscia, M.; Posteraro, F.; Micera, S. Effects of early and intensive neuro-rehabilitative treatment on muscle synergies in acute post-stroke patients: A pilot study. J. Neuroeng. Rehabil. 2013, 10, 103. [CrossRef] [PubMed]

44. Scherer, R.; Mohapp, A.; Grieshofer, P.; Pfurtscheller, G.; Neuper, C. Sensorimotor EEG patterns during motor imagery in hemiparetic stroke patients. Int. J. Bioelectromagn. 2007, 9, 155-162.

45. Rossiter, H.E.; Boudrias, M.H.; Ward, N.S. Do movement-related beta oscillations change after stroke? J. Neurophysiol. 2014, 112, 2053-2058. [CrossRef] [PubMed]

46. Alegre, M.; Labarga, A.; Gurtubay, I.G.; Iriarte, J.; Malanda, A.; Artieda, J. Beta electroencephalograph changes during passive movements: Sensory afferences contribute to beta event-related desynchronization in humans. Neurosci. Lett. 2002, 331, 29-32. [CrossRef]

47. Formaggio, E.; Storti, S.F.; Galazzo, I.B.; Gandolfi, M.; Geroin, C.; Smania, N.; Manganotti, P. Modulation of event-related desynchronization in robot-assisted hand performance: Brain oscillatory changes in active, passive and imagined movements. J. Neuroeng. Rehabil. 2013, 10, 24. [CrossRef] [PubMed]

48. Bütefisch, C.M.; Weßling, M.; Netz, J.; Seitz, R.J.; Hömberg, V. Relationship between interhemispheric inhibition and motor cortex excitability in subacute stroke patients. Neurorehabil. Neural Repair 2008, 22, 4-21. [CrossRef] [PubMed]

49. Zhuang, P.; Toro, C.; Grafman, J.; Manganotti, P.; Leocani, L.; Hallett, M. Event-related desynchronization (ERD) in the alpha frequency during development of implicit and explicit learning. Electroencephalogr. Clin. Neurophysiol. 1997, 102, 374-381. [CrossRef]

50. Gandolfi, M.; Formaggio, E.; Geroin, C.; Storti, S.F.; Boscolo Galazzo, I.; Bortolami, M.; Smania, N. Quantification of Upper Limb Motor Recovery and EEG Power Changes after Robot-Assisted Bilateral Arm Training in Chronic Stroke Patients: A Prospective Pilot Study. Neural Plast. 2018, 2018, 8105480. [CrossRef] [PubMed]

51. Page, S.J.; Fulk, G.D.; Boyne, P. Clinically important differences for the upper-extremity Fugl-Meyer Scale in people with minimal to moderate impairment due to chronic stroke. Phys. Ther. 2018, 92, 791-798. [CrossRef] [PubMed]

52. Trujillo, P.; Mastropietro, A.; Scano, A.; Chiavenna, A.; Mrakic-Sposta, S.; Caimmi, M.; Rizzo, G. Quantitative EEG for Predicting Upper Limb Motor Recovery in Chronic Stroke Robot-Assisted Rehabilitation. IEEE Trans. Neural Syst. Rehabil. Eng. 2017, 25, 1058-1067. [CrossRef] [PubMed]

53. Kitago, T.; Goldsmith, J.; Harran, M.; Kane, L.; Berard, J.; Huang, S.; Huang, V.S. Robotic therapy for chronic stroke: General recovery of impairment or improved task-specific skill? J. Neurophys. 2015, 114, 1885-1894. [CrossRef] [PubMed]

(C) 2018 by the authors. Licensee MDPI, Basel, Switzerland. This article is an open access article distributed under the terms and conditions of the Creative Commons Attribution (CC BY) license (http:/ / creativecommons.org/licenses/by/4.0/). 